Tohoku J. Exp. Med., 2004, 203, 59-63

Case Report

\title{
Warfarin-Intractable, Intraatrial Thrombogenesis in A 52-Year-Old Woman with Mitral Stenosis and Chronic Atrial Fibrillation
}

\author{
Mitsumasa Fukuchi, Koji Kumagai, Masahito Sakuma, Yutaka Kagaya, \\ Jun Watanabe, Koichi Tabayashi ${ }^{1}$ and Kunio Shirato \\ Department of Cardiovascular Medicine and ${ }^{1}$ Department of Cardiovascular \\ Surgery, Tohoku University Graduate School of Medicine, Sendai 980-8574
}

Funuchi, M., Kumagai, K., Sakuma, M., Kagaya, Y., Watanabe, J., Tabayashi, K. and Shirato, K. Warfarin-Intractable, Intraatrial Thrombogenesis in A 52-YearOld Woman with Mitral Stenosis and Chronic Atrial Fibrillation. Tohoku J. Exp. Med., 2004, 203 (1), 59-63 — Thromboembolic events are serious complications of atrial fibrillation (AF). We histologically investigated intraatrial thrombogenesis in a 52-year-old woman with mitral stenosis and chronic AF who had recurrent attacks of cerebral infarction despite continuous warfarin therapy. She underwent cardiac surgery for mitral valve replacement and maze procedure including left atrial thrombectomy. Macroscopic thrombi were found on the endocardium and their surfaces appeared rough and dark red in most areas. Histological examination showed that a single thrombus mass was composed of several tissue layers or blocks on the endocardium. Immunohistochemistry revealed stratum-like accumulations of small platelet aggregate/fibrin clot complexes in the superficial, fresh thrombus layers and multiple neovessel formation in the basal organized tissue layers. This case study suggests that intraatrial thrombi may develop in a stepwise fashion on the endocardium involving platelet aggregate/fibrin clot complex formation. - thrombosis; atrial fibrillation; immunohistochemistry; platelet; fibrin

(C) 2004 Tohoku University Medical Press

Thromboembolic events are serious complications of atrial fibrillation (AF). It has been reported that anticoagulation therapy using adjusted-dose warfarin is effective in preventing thromboembolic events in AF, with a risk reduction of 60-70\% (Atrial Fibrillation Investigators 1994; Prystowsky et al. 1996). However, even under anticoagulation therapy, the patients who have coexistent heart disease in AF still have a high risk of ischemic stroke compared to those with AF alone (Atrial Fibrillation Investigators 1994; Abe et al. 2003). We report here a woman with mitral stenosis and chronic AF who had recurrent attacks of cerebral infarction despite continuous warfarin

Received January 6, 2004; revision accepted for publication March 12, 2004.

Address for reprints: Kunio Shirato, M.D., Professor and Chairman, Department of Cardiovascular Medicine,

Tohoku University Graduate School of Medicine, 1-1 Seiryomachi, Aoba-ku, Sendai 980-8574, Japan.

e-mail: shirato@cardio.med.tohoku.ac.jp 
therapy. Using left atrial thrombectomy specimens, we histologically investigated the platelet aggregates and fibrin clot formation involved in the development of intraatrial thrombi responsible for embolic events.

\section{Case Report}

A woman had been diagnosed as having a moderate degree of mitral stenosis in AF at the age of 41 years and had been medicated with digoxin, diuretics, and adjusted-dose warfarin in Tohoku University Hospital. When she was 52 years old, she had recurrent attacks of cerebral infarction; first, right hemiparesis with aphasia and, two months later, left hemiparesis despite continuous warfarin therapy adjusted to $\approx 2.0$ of the international normalized ratio. Transesophageal echocardiography revealed the presence of several thrombi in the left atrial appendage. She underwent cardiac surgery for mitral valve replacement and maze procedure including left atrial thrombectomy. The thrombectomy specimens were immediately fixed in $4 \%$ paraformaldehyde for histological examination. In serial cryostat sections, immunohistochemistry was performed to localize platelets (von Willebrand factor or glycoprotein IIb/IIIa), fibrin clots (fibrin), and endothelial cells (CD31) using the ABC peroxidase or alkaline phosphatase methods as previously described (Fukuchi et al. 2001). Immunoreactive sites were visualized with $0.025 \%$ 3,3-diaminobenzidine and $0.01 \%$ hydrogen peroxide or with Vector Red alkaline phosphatase substrate kit (Vector Lab., Burlingame, CA, USA). Tissue collection was performed with permission of the patient together with informed consent, and this study was approved by the Ethics Committee of Tohoku University.

\section{Pathological Findings}

Macroscopic thrombi were found on the endocardium of the left atrial appendage. The surfaces of thrombi appeared rough and dark red in most areas. Fig. 1a shows a cut section of a beansized, isolated thrombus that could have caused a future stroke. This thrombus mass was composed of several tissue layers or blocks on the endocardium (Fig. 1b). Immunohistochemistry showed heterogeneous cellular and noncellular compositions among different layers or blocks of the same thrombus mass. In the superficial layers, platelet aggregates (positive to von Willebrand factor or glycoprotein IIb/IIIa) and fibrin clots (positive to fibrin) were distributed with a stratum-like appearance (Figs. 1c and 1d). Higher magnification (right panels) showed scattered small particles or plates of platelet aggregates and a framework structure of fibrin clots. Double immunohistochemistry revealed that each platelet aggregate core was wrapped by a fibrin clot framework (Fig. 1e). On the other hand, multiple neovessel formation (positive to CD31) but no platelet aggregate/ fibrin clot was observed in the basal organized tissue layers (Figs. 1f and 1g). Intermediate layers of the thrombus mass were mixed with platelet aggregate/fibrin clots and neovessel formation.

\section{Discussion}

This case report provides an immunohistochemical analysis of intraatrial thrombi in a woman with mitral stenosis and chronic AF who had recurrent attacks of cerebral infarction despite continuous warfarin therapy. Macroscopic thrombi in the left atrial appendage had heterogeneous compositions ranging from organized tissue layers to fresh thrombus layers. In the fresh thrombus layers, both platelet aggregates and fibrin clots were distributed with a stratum-like appearance. Double immunohistochemistry revealed that each platelet aggregate core was wrapped by a fibrin clot framework. Our observations are noteworthy on several points.

First, we probably characterized the stasisinduced thrombogenesis in the left atrial appendage by histological analysis. Recent echocardiographic studies reported that left atrial spontaneous echo contrast or thrombi were frequently found in patients with underlying heart disease in $\mathrm{AF}$ and were associated with a reduction or lack of active left atrial appendage flow (Gonzalez- 

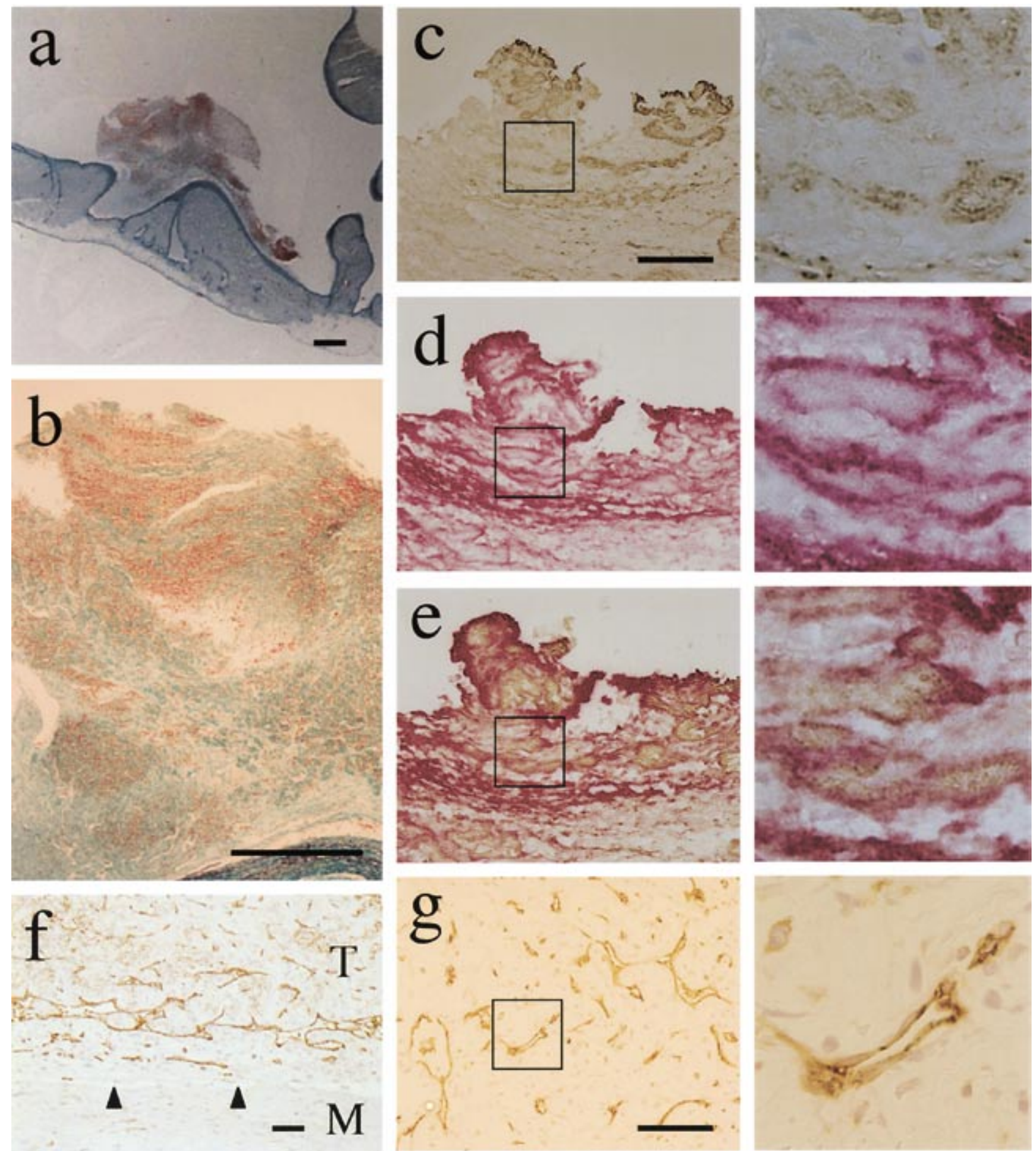

Fig. 1. Immunohistochemical analyses of platelet aggregates and fibrin clot formations in left atrial thrombectomy specimens obtained from a 52-year-old woman with mitral stenosis and chronic atrial fibrillation. $\mathbf{a}$ and $\mathbf{b}$ : Elastica-Masson staining in a cryostat section. Bar=1 mm. c-g: Immunohistochemistry was performed in serial sections using the ABC peroxidase method for von Willebrand factor (c) and CD31 (f and $\mathbf{g}$ ), the ABC alkaline phosphatase method for fibrin (d), and double immunohistochemistry for von Willebrand factor and fibrin (e). The box in the left panel (c-e and $\mathbf{g}$ ) shows the tissue area magnified in the right panel. Arrowheads indicate the endocardial surface. T, thrombus; M, myocardium. Bar $=100 \mu \mathrm{m}$. 
Torrecilla et al. 2000; Narumiya et al. 2003). In such flow conditions, thrombus growth on the endocardium may easily occur resulting in a macroscopic mass formation as shown in Fig. 1a. Second, heterogeneous compositions ranging from organized tissue layers to fresh thrombus layers within the same thrombus mass (Fig. 1b) indicate that thrombus growth on the endocardium proceeded slowly in a stepwise fashion. Since our patient had received full-dose warfarin therapy until an attack of cerebral infarction, our findings may not be applicable to the thrombogenesis that occurs within a few days after acute AF attack (Stoddard et al. 1995). This stepwise thrombus growth also suggested that the previous thrombus formation on the endocardium had become a locus in which fresh thrombi developed. Third, fresh thrombus layers showed a stratum-like appearance consisting of abundant accumulations of a small platelet aggregate core wrapped by a fibrin clot framework (Fig. 1e). This platelet aggregate/fibrin clot complex may be a single unit of the thrombus growth. This is the first report that histologically demonstrates the interplay between platelet aggregates and fibrin clots in intraatrial thrombi, although increased plasma levels of both fibrin D-dimer and platelet beta-thromboglobulin have been shown in AF patients (Lip et al. 1996). In contrast to our findings, thrombogenesis at a high shear rate has been experimentally characterized by localizations of fibrinogen in the inner areas and platelet thrombi mediated through von Willebrand factor at the outer surfaces (Matsui et al. 2002).

By using left atrial thrombectomy specimens from a woman with mitral stenosis and chronic $\mathrm{AF}$, we demonstrated that warfarin-intractable, intraatrial thrombogenesis occurred in a stepwise fashion on the endocardium involving platelet aggregate/fibrin clot complex formation. This case study provides histological evidence suggesting that, compared with anticoagulation therapy alone, its combination with antiplatelet therapy may be further effective in preventing intraatrial thrombus growth in AF patients with a high risk of ischemic stroke.

\section{Acknowledgements}

We thank Mr. Brent Bell for reading the manuscript.

\section{References}

Abe, Y., Asakura, T., Sakamoto, N., Ishikawa, S., Muroi, S., Saitoh, F., Satoh, M., Suzuki, S., Ono, M., Sakabe, A., Iwai, M., Sando, M., Gotou, J., Watanabe, Y., Nagata, K., Maehara, K. \& Maruyama, Y. (2003) Embolic attack in patients with atrial fibrillation and atrial thrombus depends on the character of the thrombus. Circ. J., 67, 203-208.

Atrial Fibrillation Investigators (1994) Risk factors for stroke and efficacy of antithrombotic therapy in atrial fibrillation. Analysis of pooled data from five randomized controlled trials. Arch. Intern. Med., 154, 1449-1457.

Fukuchi, M., Watanabe, J., Kumagai, K., Katori, Y., Baba, S., Fukuda, K., Yagi, T., Iguchi, A., Yokoyama, H., Miura, M., Kagaya, Y., Sato, S., Tabayashi, K. \& Shirato, K. (2001) Increased von Willebrand factor in the endocardium as a local predisposing factor for thrombogenesis in overloaded human atrial appendage. J. Am. Coll. Cardiol., 37, 1436-1442.

Gonzalez-Torrecilla, E., Garcia-Fernandez, M.A., Perez-David, E., Bermejo, J., Moreno, M. \& Delcan, J.L. (2000) Predictors of left atrial spontaneous echo contrast and thrombi in patients with mitral stenosis and atrial fibrillation. Am. J. Cardiol., 86, 529-534.

Lip, G.Y.H., Lip, P.L., Zarifis, J., Watson, R.D.S., Bareford, D., Lowe, G.D.O. \& Beevers, D.G. (1996) Fibrin D-dimer and $\beta$-thromboglobulin as markers of thrombogenesis and platelet activation in atrial fibrillation. Effects of introducing ultra-low-dose warfarin and aspirin. Circulation, 94, 425-431.

Matsui, H., Sugimoto, M., Mizuno, T., Tsuji. S., Miyata, S., Matsuda, M. \& Yoshioka, A. (2002) Distinct and concerted functions of von Willebrand factor and fibrinogen in mural thrombus growth under high shear flow. Blood, 100, 3604-3610.

Narumiya, T., Sakamaki, T., Sato, Y. \& Kanmatsuse, K. (2003) Relationship between left atrial appendage function and left atrial thrombus in patients with nonvalvular chronic atrial fibrillation and atrial flutter. Circ. J., 67, 68-72. 
Prystowsky, E.N., Benson, D.W., Jr., Fuster, V., Hart, R.G., Kay, G.N., Myerburg, R.J., Naccarelli, G.V. \& Wyse, D.G. (1996) Management of patients with atrial fibrillation. A statement for Healthcare Professionals from the Subcommittee on electrocardiography and electrophysiology, American Heart Association. Circulation,
93, 1262-1277.

Stoddard, M.F., Dawkins, P.R., Prince, C.R. \& Ammash, N.M. (1995) Left atrial appendage thrombus is not uncommon in patients with acute atrial fibrillation and a recent embolic event: a transesophageal echocardiographic study. J. Am. Coll. Cardiol., 25, 452-459. 